\title{
Endovascular implantation of iodine-125 seed strand combined and stent placement for locally advanced pancreatic ductal adenocarcinoma with vascular invasion: a prospective single-arm pilot study
}

\author{
Linlin Wu, MD', Yanbo Zhang, MM², Zihan Zhang, MD³, Fengfeng Li, MM, Jianjun Luo, MD³ \\ 'Department of Oncology, Tengzhou Central People's Hospital, Tengzhou City, Shandong Province, China, ${ }^{2}$ Department of Pharmacy. \\ Tengzhou Central People's Hospital, Tengzhou City. Shandong Province, China, ${ }^{3}$ Department of Interventional Radiology, Affiliated \\ Zhongshan Hospital of Fudan University, Xuhui District, Shanghai, China, ${ }^{4}$ Department of Neurosurgery, Tengzhou Central People's Hospital \\ Tengzhou City, Shandong Province, China
}

\begin{abstract}
Purpose: To investigate the safety and feasibility of endovascular brachytherapy using iodine-125 ( $\left.{ }^{125} \mathrm{I}\right)$ seed strand for locally advanced pancreatic ductal adenocarcinoma (PDAC) with vascular invasion.

Material and methods: From January 2010 to January 2015, 12 patients diagnosed with locally advanced, inoperable PDAC with splenic or superior mesenteric vein invasion were enrolled in the present study and received endovascular brachytherapy combined with regional intra-arterial infusion chemotherapy. Standardized software was used for dose calculation. Procedure-related and radiation complications were documented and assessed. Overall survival was calculated with the Kaplan-Meier approach.

Results: The technical success rate of ${ }^{125}$ I seed strand implantation and stent placement was $100 \%$. During followup with a mean duration of $17.00 \pm 6.07$ months (range, 6 24 months), the mean and median survival times were 12.0 \pm 2.4 months (95\% CI: 7.4 16.6 months) and $10.7 \pm 1.4$ months (95\% CI: 8.0 13.5 months), respectively. One month after the treatment, the disease control and objective rates were $83.8 \%$ and $58.3 \%$, respectively. The $6-$, $12-$, and $15-$ month cumulative survival rates were $66.7 \%, 47.6 \%$, and $9.5 \%$, respectively.

Conclusions: Endovascular brachytherapy using ${ }^{125} \mathrm{I}$ seed strand and stent placement may be a safe and effective treatment option for locally advanced pancreatic duct adenocarcinoma with vascular invasion.

J Contemp Brachytherapy 2020; 12, 5: 427-434 DOI: https://doi.org/10.5114/jcb.2020.100375
\end{abstract}

Key words: pancreatic ductal adenocarcinoma, ${ }^{125} \mathrm{I}$ seed strand, vascular invasion.

\section{Purpose}

Pancreatic ductal adenocarcinoma (PDAC) is one of most lethal known malignancy, with a 5-year survival rate of less than $7 \%[1,2,3]$. Over $85 \%$ of patients with PDAC present with locally advanced disease or distant metastases, which preclude radical surgical treatment [4]. Conventional chemotherapy is limited by its systemic side effects and poor patient tolerance. As mortality increases after invasive procedures, more aggressive radiotherapy is occasionally indicated. As an example, radiotherapy with external beam, intraluminal brachytherapy, or interstitial brachytherapy has been found to be effective in improving local control in patients with advanced pancreatic cancer $[5,6,7]$. Yang et al. reported that in patients with PDAC, complicated by obstructive jaundice, the recurrence of obstructive jaundice significantly decreased and the overall survival was extended after intraluminal brachytherapy using iodine-125 ( $\left.{ }^{125} \mathrm{I}\right)$ seed strand [8].

Not only biliary obstruction, but a large proportion of patients are diagnosed with vascular invasion [9]. Recently, endovascular brachytherapy using ${ }^{125}$ I seed strand was reported to be safe and effective for hepatocellular carcinoma with portal vein tumor thrombus $[10,11]$. Based on these results, the potential of endovascular brachytherapy using ${ }^{125} \mathrm{I}$ seed strand in controlling PDAC invasion was investigated in this study as well as evaluating the safety and feasibility of endovascular brachytherapy for locally advanced PDAC with vascular invasion.
Address for correspondence: Jianjun Luo, Department of Interventional Radiology, Affiliated Zhongshan Hospital of Fudan University, No. 180 Fenglin Road, Xuhui District, Shanghai 200032, China,

phone/fax: 86021-64041990-2922, ఐ e-mail: shljj1971@163.com
Received: 24.03 .2020

Accepted: 03.07 .2020

Published: 30.10 .2020 


\section{Material and methods}

\section{Patients}

This prospective single-arm pilot study was approved by the ethics committee and institutional review board of Tengzhou Central People's Hospital. In total, 20 Chinese patients with advanced PDAC with splenic vein (SV) and superior mesenteric vein (SMV) invasion, who were admitted to Tengzhou Central People's Hospital between January 2010 and January 2015 were enrolled. Upon admission, endovascular brachytherapy (EVBT) using ${ }^{125} \mathrm{I}$ combined with regional intra-arterial infusion chemotherapy (RIAC) or transarterial chemoembolization (TACE) was recommended by attending physicians. Among 20 enrolled patients, 12 accepted the EVBT-TAI or TACE treatment.

Patients were included with the following criteria: 1. Locally advanced inoperable PDAC with vascular invasion; 2. Eastern Cooperative Oncology Group (ECOG) performance status 0-2; and 3. Adequate hematological, liver, and renal function. PDAC was diagnosed by clinical and radiological examinations as well as histopathologic results after biopsy. Excluded were patients with ECOG performance status $>2$, any contraindication to a transhepatic puncture procedure, such as compromised coagulative capability (platelet count $<50 \times 10^{9} / 1$ or prothrombin activity $<50 \%$ ), renal failure, cardiac ejection fraction $<50 \%$, or end-stage diseases as well as patients who were not cooperative during the procedure.

Before ${ }^{125}$ I seeds implantation, and RIAC or TACE procedures, the benefits and potential adverse events related to these techniques were explained thoroughly to eligible

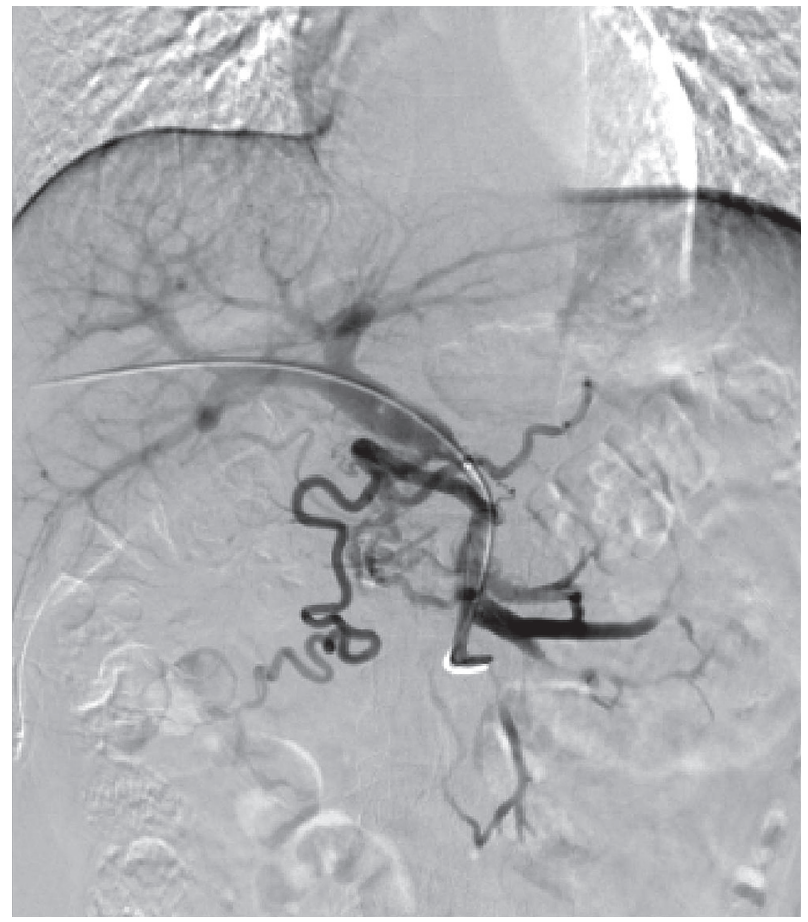

Fig. 1. Images of iodine-125 ( $\left.{ }^{125} \mathrm{I}\right)$ seed strand implantation in a 50-year-old man. The second-order branch of the left portal vein was catheterized patients and their family members. All patients submitted written informed consent before the start of therapy.

\section{Design}

Contrast-enhanced computed tomography (CT) or magnetic resonance imaging (MRI) was performed and subsequently analyzed by a multidisciplinary cancer specialist group for disease staging. The primary endpoints included overall survival (OS) and time to progression (TTP). Complications were documented and assessed according to the Common Terminology Criteria for Adverse Events, 4.0 criteria. The overall survival was calculated as the interval between ${ }^{125}$ I seed strand implantation and patient's death.

\section{Iodine-125 seed strand}

Model $6711^{125}$ I seed (XinKe, Shanghai, China) was used in this study as a cylindrical brachytherapy source, with an active length of $3.25 \mathrm{~mm}$ encapsulated by titanium. The diameter and length of titanium capsule was 0.8 and $4.5 \pm 0.5 \mathrm{~mm}$, respectively. Radioactivity of each ${ }^{125}$ I seed was $25.9 \mathrm{MBq}$, with a half-life of 59.4 days. The principal photon emissions were 27.4, $31.4 \mathrm{keV}$ X-ray, and $35.5 \mathrm{keV}$ Gamma-ray, respectively. Half-value thickness of tissue for ${ }^{125} \mathrm{I}$ seed was $17 \mathrm{~mm}$, and the incipient dose rate was $7 \mathrm{cGy} / \mathrm{h}$.

\section{Intra-SV or SMV implantation of stent and ${ }^{125}$ I seed strand}

The second-order branch of intrahepatic portal vein was punctured with a 22-gauge Chiba needle (Cook Inc., Bloomington, Indiana, USA) under ultrasound guidance in patients receiving the therapy. When access was confirmed, a 0.018-inch wire (Cook Inc.) was inserted into the portal vein. A 6-F NEFF set and a 0.035 -inch, $150 \mathrm{~cm}$-long wire (Terumo, Tokyo, Japan) combined with a 4-F Cobra catheter (Cordis, Miami Lakes, Florida, USA) were then manipulated across the obstructed SV or SMV. The Cobra catheter was removed, and the outer cannula of NEFF set was replaced by a 7-F, $23 \mathrm{~cm}$-long sheath (Cordis) over the wire. Portography was performed to measure the diameter and length of obstructed SV or SMV by a 5-F calibrated pigtail catheter (Cook Inc.). The pigtail catheter was positioned at the distal part of SV or SMV for pressure measurement. The number of ${ }^{125} \mathrm{I}$ seeds planned to be implanted was calculated using the following formula: $\mathrm{N}=$ length of obstructed SV or SMV $(\mathrm{mm}) / 4.5+4$. These seeds were arranged linearly and sealed into a 4-F catheter continuously to construct a ${ }^{125} \mathrm{I}$ seed strand. Then, $50 \mathrm{U} / \mathrm{kg}$ of heparin (XingYi, Shanghai, China) was administered intravenously and two 0.035 -inch, $260 \mathrm{~cm}$ long stiff wires (Terumo) were inserted into the SMV through a 7-F sheath. After the sheath was removed, the outer cannula of NEFF set and self-expendable stent with appropriate size were introduced to the SV or SMV over one of the stiff wires, respectively. Stent was deployed from the distal to the proximal part of the SV or SMV. Through the outer cannula of NEFF set, the ${ }^{125} \mathrm{I}$ seed strand was delivered to the target position and released 

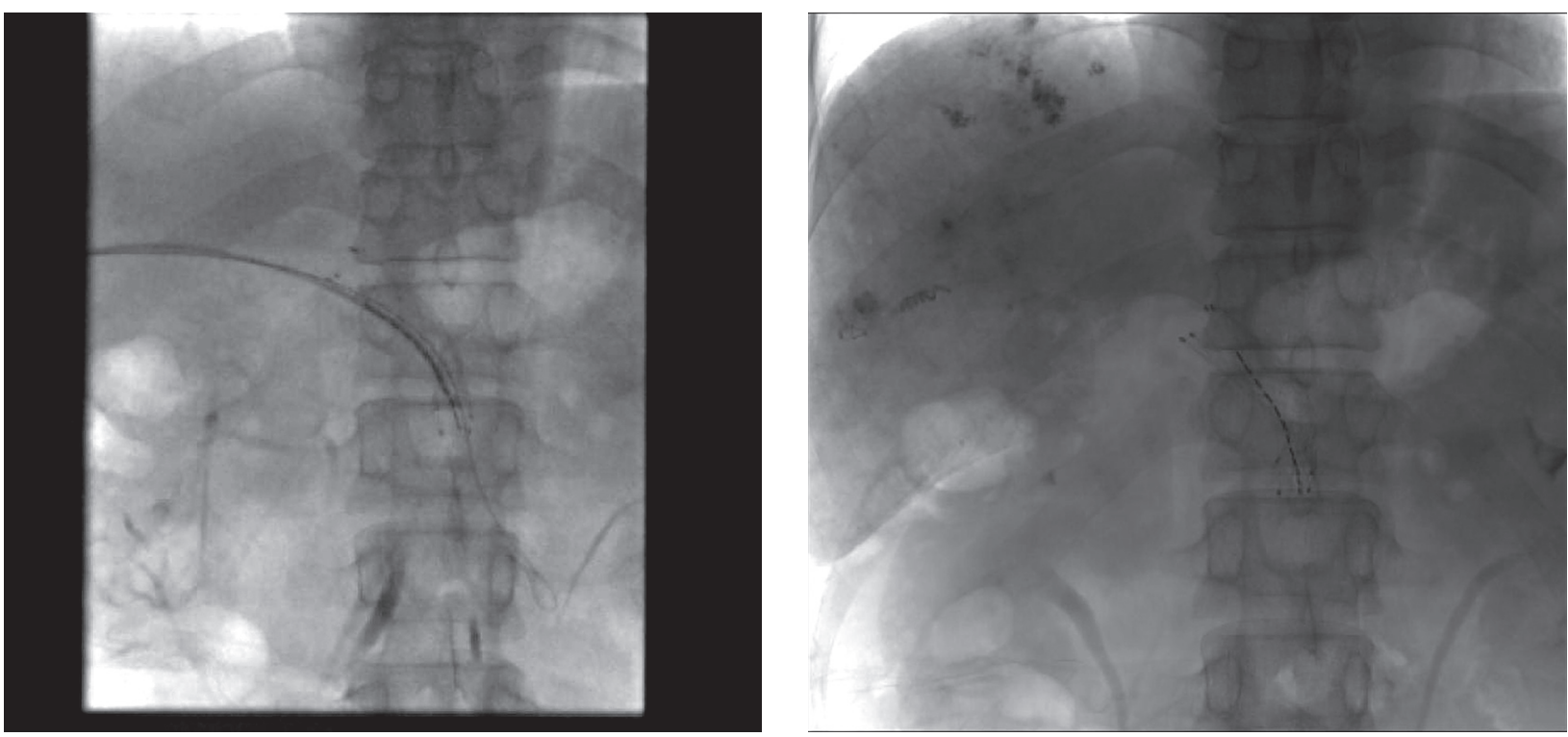

Fig. 2. A 5-F calibrated pigtail catheter was placed in SMV. Tumor thrombus in the proximal part of SMV was clearly shown on portography. A $12 \times 80-\mathrm{mm}$ self-expandable stent (black arrow) and ${ }^{125}$ I seed strand (white arrow) with 20 seeds loaded were placed precisely in the obstructed SMV. ${ }^{125}$ I seed strand was fixed steadily between the stent and MPV

between the stent, SV, or SMV. Repeated portography and pressure measurement were performed before the transhepatic puncture track was occluded by coils (Cook Inc.) (Figures 1-3).

\section{RIAC preparation}

Before the procedure, a CT scan of pancreas was performed, and the image was transmitted to the radioactive particle TPS computer to reconstruct the three-dimensional digital image and delineate the target area. According to the three mutually perpendicular diameters of tumor target volume, the matched peripheral dose (MPD) and particle radioactivity of the tumor were calculated, along with the required number of particles and the spatial distribution of particles. Final determination of the position, direction, and number of implanted guide pins were performed.

\section{RIAC and TACE procedures}

RIAC was performed in all patients under local anesthesia. Right femoral artery puncture and intubation were done with 5-Fr arterial sheath. Meanwhile, an angiography of celiac and superior mesenteric arteries was carried out with 4- or 5-Fr catheters. Gemcitabine was given at a dose of $1000 \mathrm{mg} / \mathrm{m}^{2}$, followed by oxaliplatin of $100 \mathrm{mg} / \mathrm{m}^{2}$. In patients with lesions located in the head of pancreas, one third of the drug was infused via the superior mesenteric artery, and the other two thirds through the gastroduodenal artery. In patients with lesions situated in the pancreatic body or tail, the great pancreatic, caudal pancreatic, and dorsal pancreatic arteries could all contribute to the blood supply of tumor. Therefore, candidate arteries for infusion were carefully evaluated before every procedure. Alternative methods were considered if necessary.
A full dose of chemotherapeutic agent was infused through the splenic artery if the afore-mentioned arteries originated from this artery. Chemotherapeutic agent was infused via the celiac artery if feeding arteries of tumor originated from the common hepatic or celiac artery. One third of the drug was given through the superior mesenteric artery and two thirds via the splenic artery if the superior mesenteric artery contributed to the tumor blood supply. In the last case, if feeding arteries of the tumor could be directly super-selected, the drug was infused through these feeding arteries using 3-Fr catheters.

Five patients were diagnosed with hepatic metastases and subsequently underwent both RIAC and TACE simultaneously. To identify all feeding arteries of the tumor as distally as possible, angiography of the celiac,

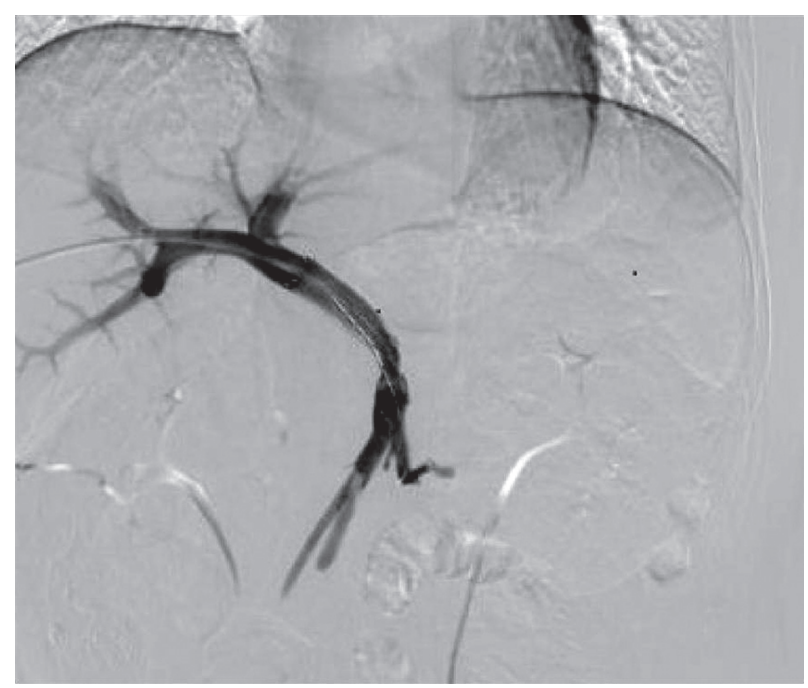

Fig. 3. The blood flow through patent stent from the distal MPV to the right portal vein was shown on portography 
hepatic, superior mesenteric, left gastric, and bilateral inferior phrenic arteries were performed by catheterization with a 2.7-F microcatheter (Renegade, Boston Scientific, Natick, Massachusetts, USA). Using a pumping method, $10-50 \mathrm{mg} / \mathrm{m}^{2}$ of epirubicin (Pharmorubicin, Pfizer, New York, USA) was mixed with a 5-20 ml of iodized oil (Lipiodol Ultra Fluide, Laboratoire Guerbet, Aulnay-sous-Bois, France). The doses of epirubicin and iodized oil were determined based on patient's body weight and tumor vascularity, respectively. Under fluoroscopic monitoring, the mixture was infused at a rate of $0.5-1 \mathrm{ml} / \mathrm{min}$ through microcatheter until static flow in tumor feeding vessels was achieved. Finally, gelatin sponge (Jingling, Jiangsu, China) was used to embolize the feeding artery of tumor.

\section{Post-procedure management and evaluation}

After the procedure, all patients received intravenous tropisetron hydrocloride $(5 \mathrm{mg} / \mathrm{d}$, Novartis Pharma Schweiz AG, Switzerland), omeprazole (40 mg/d, Changzhou Pharmaceutics, Jiangsu, China), and ornithine aspartate (10 g/d, Merz Pharma, GmbH \& Co. KGaA, Frankfurt, Germany) for 3-5 days. Pain and fever attributed to post-embolization syndrome were controlled individually with non-steroidal anti-inflammatory drugs or opioids. Subcutaneous low-molecular-weight heparin (XinYi, Shanghai, China) of $4100 \mathrm{U}$ was given twice a day. One day after the procedure, all RIAC patients underwent single photon emission computer tomography combined with CT scan (SPECT/CT) to evaluate the distribution of radiation from implanted ${ }^{125} \mathrm{I}$ seed strands. Three days after the procedure, all patients were started on warfarin (XinYi, Shanghai, China), with an initial dose of $2.5 \mathrm{mg}$ per day and continued for 6 months. The dose of warfarin was adjusted based on a coagulability test, with an international normalized ratio goal of 1.5-1.8.

\section{Follow-up and repeated TACE}

The follow-up period was defined as the duration from the date of entry into treatment to death or last follow-up date of $1^{\text {st }}$ March 2016. All patients were followed-up every 30-45 days using contrast-enhanced CT or MRI to evaluate tumor response to treatment. At follow-up, laboratory tests were performed to evaluate patients' liver and renal function, blood cell count, and coagulative function. Repeated RIAC or TACE with the same protocol was performed, when residual tumor with enhancement on arterial phase, occurrence of a new lesion, or both were observed.

\section{Efficacy and safety assessment}

According to the modified response evaluation criteria in solid tumor (mRECIST) recommended by the American Association for the Study of Liver Diseases [12], the response of PDAC to RIAC and hepatic metastases were classified as complete response (CR), partial response (PR), stable disease (SD), and progressive disease (PD). An objective response was defined as $\mathrm{CR}+\mathrm{PR}$, and disease control was defined as $\mathrm{CR}+\mathrm{PR}+\mathrm{SD}$. The treatment responses of these patients were assessed using the best tumor response rating of disease control, which maintained for at least 4 weeks from the first manifestation of mRECIST.

Primary endpoints included overall survival (OS) and TTP. The survival time was determined as the period from the day of procedure to patients' death, or to their last follow-up. The occurrence of events, such as radiological progression, obstructive jaundice, and variceal bleeding was recognized as disease progression of PDAC. Therefore, TTP was the time from therapy to either the occurrence of above-mentioned events or patient's death.

Laboratory values obtained 180 days after ${ }^{125} \mathrm{I}$ seed strand placement were used for toxicity analysis. This interval was chosen, because the half-life of ${ }^{125}$ I seed used in this study was 59.4 days, which means that about $87.5 \%$ of radiation energy of ${ }^{125}$ I seed would be released within 180 days after the implantation. Likewise, most acute radiation toxicities possibly related to ${ }^{125}$ I seed implantation would occur during this interval. Toxicity occurring beyond this time window was not considered to be treatment-related.

In all enrolled patients, the RIAC- and TACE-related toxicities were assessed using the Common Terminology Criteria for Adverse Events (CTCAE) v.4.0. Adverse events that occurred within 4 weeks after RIAC and TACE were documented. Symptoms of post-embolization syndrome, such as abdominal pain, fever with unknown origin, nausea, and vomiting were expected and therefore not documented separately.

\section{Statistical analysis}

Continuous variables were expressed as mean \pm SD. Paired-sample $t$-test was applied to evaluate laboratory test changes. A $p$ value less than 0.05 indicated a significant difference. OS and TTP were calculated using Kaplan-Meier method. All data analyses were conducted with a statistical software (SPSS, version 22.0, IBM, USA).

\section{Results}

\section{Baseline characteristics of patients and tumors}

A total of 12 patients with advanced PDAC that invaded the SV and SMV underwent EVBT-stent-RIAC or TACE. There were 8 males $(66.7 \%)$ and 4 females $(33.3 \%)$, with a mean age of $56.2 \pm 5.0$ years (range, $47-63$ years). Four patients were diagnosed with pancreatic head carcinoma, and 8 patients with pancreatic body or tail carcinoma. Tumor invasion to the SV or SMV was found in 4 patients. According to NCCN clinical staging guidelines in oncology for pancreatic adenocarcinoma (v. from 2011), 6 patients presented phase III and the other 6 patients had phase IV tumors. Hepatic and adrenal metastases were found in 5 and 1 patients, respectively. The baseline characteristics of these patients and tumors are presented in Table 1.

\section{Stent and ${ }^{125}$ I seed strand placement}

The technical success rates of stent placement and ${ }^{125} \mathrm{I}$ seed strand placement were both $100 \%$. No complication, such as intraperitoneal hemorrhages, infection, biliary fistula, and intestinal fistula was observed. The mean length 
Table 1. Patients characteristics

\begin{tabular}{llllllcc} 
Case No. & Sex & Age & Location & Staging & Intravenous & $\begin{array}{c}\text { Obstruction } \\
\text { length }\end{array}$ & $\begin{array}{c}\text { Number of } \\
\text { particles }\end{array}$ \\
\hline 1 & M & 62 & Head & III & SMV & 3 & 10 \\
\hline 2 & M & 58 & Body & VI & SV & 3.5 & 12 \\
\hline 3 & M & 57 & Body & III & SV & 4 & 14 \\
\hline 4 & F & 60 & Head & VI & SMV & 3 & 10 \\
\hline 5 & M & 48 & Body & VI & SV & 4 & 16 \\
\hline 6 & M & 47 & Body & III & SV & 5 & 20 \\
\hline 7 & F & 67 & Head & VI & SMV & 3 & 12 \\
\hline 8 & M & 54 & Body & III & SMV & 4 & 14 \\
\hline 9 & F & 55 & Head & VI & SMV & 3 & 12 \\
\hline 10 & M & 51 & Body & III & SV & 6 & 22 \\
\hline 11 & F & 52 & Body & VI & SV & 5 & 18 \\
\hline 12 & M & 55 & Body & III & SV & 4 & 15
\end{tabular}

$S M V$ - superior mesenteric vein, $S V$ - splenic vein, $M$ - male, $F$-female

of obstructed SV or SMV was $38 \mathrm{~mm}$ (range, 20-50 mm). The mean number of ${ }^{125} \mathrm{I}$ seed implanted in a patient was $14.0 \pm 3.8$ seeds (range, 10-22 seeds). After the first therapy, radiation dose was verified by the TPS (Hejia China).

\section{SPECT/CT scan}

SPECT/CT scans one day after the procedure showed that all stents and ${ }^{125}$ I seed strands were correctly placed in the obstructed SV or SMV without displacement. Radiation emitted by the ${ }^{125}$ I seed strand was distributed homogeneously, which presented as a cylindrical shape, and completely covered the target lesion in SPECT/CT scan.

\section{Overall survival}

During follow-up with a mean duration of $17.00 \pm 6.07$ months (range, 6-24 months), 11 (91.7\%) out of 14 patients died. The mean and median survival times were $12.0 \pm 2.4$ (95\% CI: 7.4 16.6 months) and $10.7 \pm 1.4$ months (95\% CI: 8.0 13.5 months), respectively (Figure 4). The 6-, 12-, and 15 -month cumulative survival rates were $66.7 \%, 47.6 \%$, and $9.5 \%$, respectively. Among dead patients, $6(54.6 \%)$ had a liver failure, $3(27.3 \%)$ had an obstructive jaundice, and 1 patient $(9.1 \%)$ suffered from a lung metastasis.

\section{Response of PDAC}

During the study, the average number of RIAC performed for each patient was $3 \pm 1.2$ (range, 1-5), and the average number of TACE performed for each patient was $3 \pm 5.3$ (range, 1-4). The mean dosages of gemcitabine and oxaliplatin used in RIAC were $29.4 \pm 9.4 \mathrm{mg}$ (range, $10-50 \mathrm{mg}$ ) and $9.4 \pm 4.2 \mathrm{ml}$ (range, 3-15 ml), respectively. Assessment of the tumor response to treatment using mRECIST criteria [12] indicated that CR, PR, SD, and PD were achieved in $0(0 \%), 7(58.3 \%), 3(25.0 \%)$, and $2(16.7 \%)$ patients, respectively. The disease control rate was $83.3 \%$ and the objective response rate was $58.3 \%$ (Figures 5-7). Volume and activity of tumors significantly decreased after the procedure, as demonstrated by PET/CT images (Table 2).

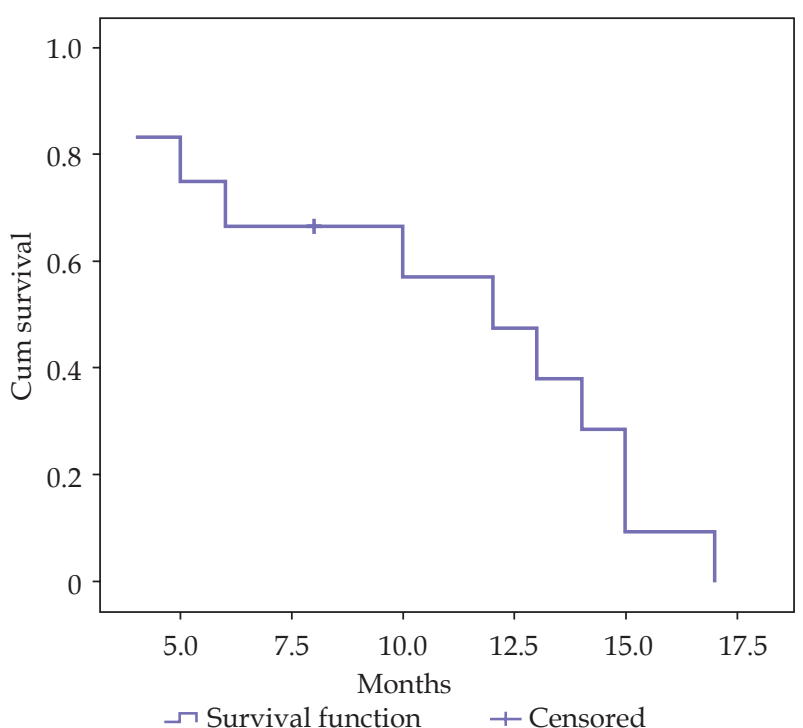

Fig. 4. Kaplan-Meier method analyzing the overall survival of patients (survival function diagram). Temporal interval $-17.00 \pm 6.07$ months, Case load $-n=14$, Confidence interval - 95\% CI, Statistical software - SPSS 21.0 (SPSS, Inc., Chicago, IL, USA)

\section{Procedure-related adverse events}

No serious procedure-related complications, such as intraperitoneal bleeding, acute hepatic failure, or radiation enteritis were observed during the study. The incidence of post-chemotherapy syndromes, including nausea and vomiting was documented. Among all enrolled patients, $3(36 \%)$ suffered from a fever and $1(8.3 \%)$ had an abdominal pain, which could be related to chemoembolization, and recovered 3-5 days after the procedure following symptomatic treatment. Thirty days after the procedure, laboratory tests of all patients recovered to the pre-therapy level (Table 3). No grade 3 or 4 treatmentrelated adverse events assessed by the National Cancer Institute's Common Terminology Criterial (version 4.0) [13] were observed. 

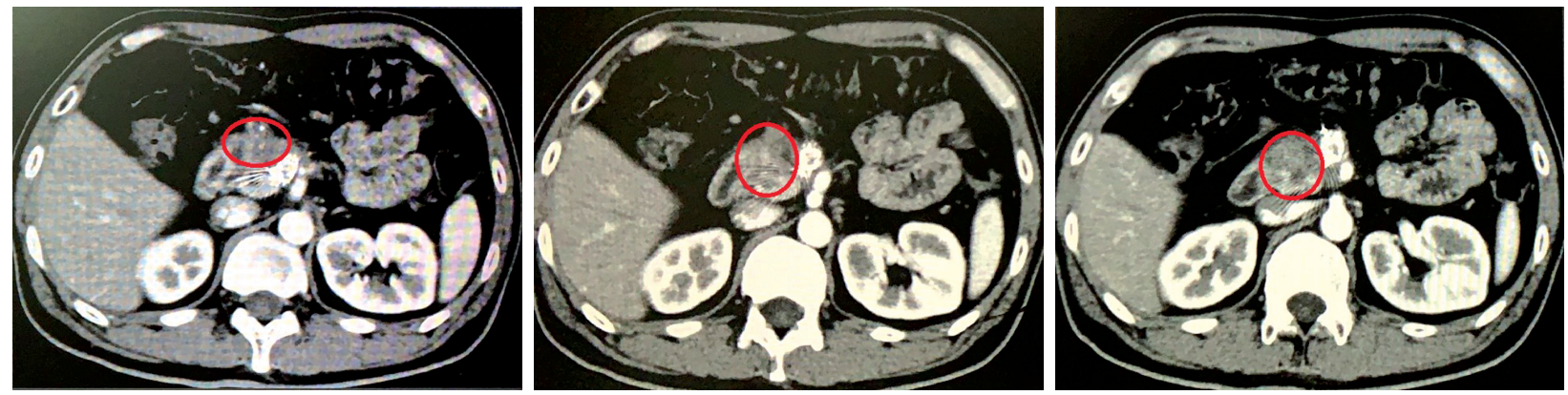

Fig. 5. Contrast-enhanced abdominal CT images at 1 month after the first therapy. Red circle marks significantly decreased tumor volume and the patency of stent in SMV
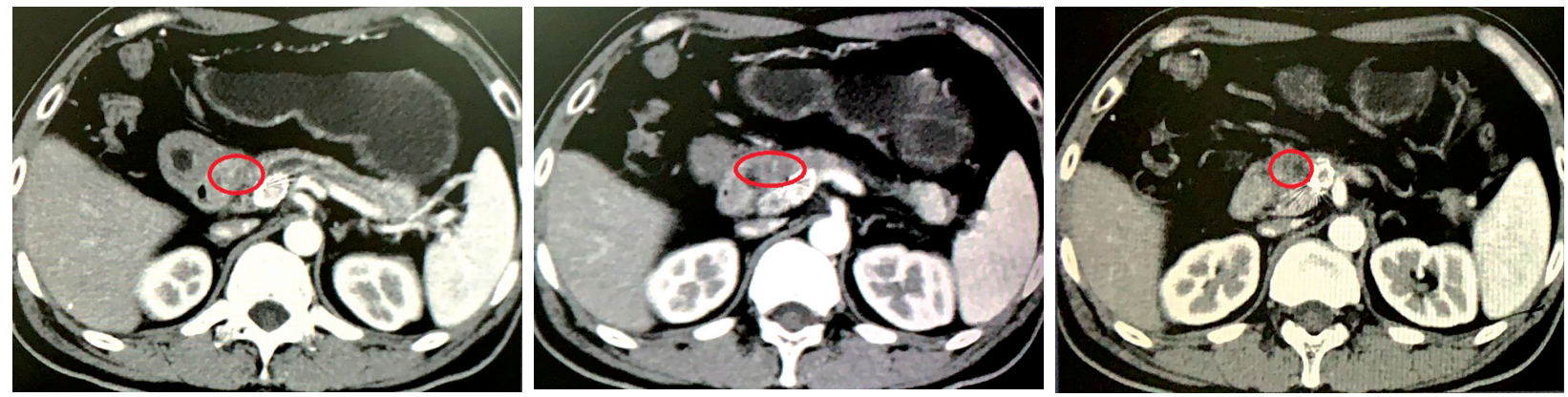

Fig. 6. Contrast-enhanced abdominal CT images at 2 months after the first therapy
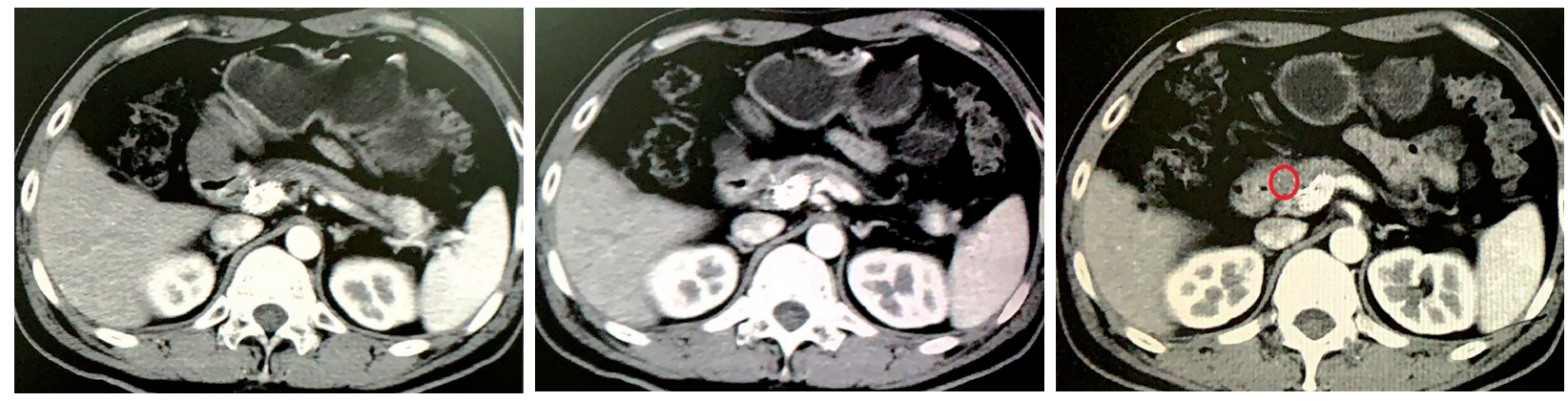

Fig. 7. Contrast-enhanced abdominal CT images at 3 months after the first therapy

Table 2. Contrast of volume and activity of tumors before and after the procedure

\begin{tabular}{lccc} 
& Early phase SUV & Lag phase SUV & $\begin{array}{c}\text { Shortest } \\
\text { diameter }\end{array}$ \\
\hline Before & $4.04 \pm 0.22$ & $5.85 \pm 0.23$ & $3.48 \pm 0.33$ \\
\hline After & $3.26 \pm 0.17$ & $4.69 \pm 0.25$ & $3.08 \pm 0.32$ \\
\hline$t$ & 4.29 & 3.74 & 2.61 \\
\hline$p$ & 0.001 & 0.003 & 0.024
\end{tabular}

\section{Discussion}

The prognosis of PDAC, with its largely unknown pathogenesis, remains suboptimal. Only about $15 \%$ of patients with this disease are the right candidates for $\mathrm{cu}-$ rative surgical resection, and the other $85 \%$ of patients would present with phase III or IV of the disease at their first medical visit [14]. Tumor at the pancreatic head is likely to cause obstructive jaundice, while tumor at the body and tail of pancreas is prone to invade the SV and
SMV. Such complications are contributing further to poor prognosis of the disease. Currently, comprehensive therapy (radiotherapy plus chemotherapy) is the most commonly adopted treatment modality for locally advanced pancreatic carcinoma [15]. However, the role of comprehensive therapy is limited by its potential systemic side effects and drug insensitivity [16].

Currently, RIAC is the most common interventional approach for PDAC, and lesions with rich blood supply can be treated by RIAC combined with transarterial embolization. However, despite comprehensive treatments with such therapies, the improvement in overall survival remains largely limited [17]. Endovascular and interstitial brachytherapies have been found to be effective when used as palliative therapy to improve local control in patients with locally advanced pancreatic cancer.

Many studies have proved that in current radiation therapy, ${ }^{125}$ I seed-based low-dose-rate (LDR) radiotherapy presents better efficacy and safety than high-dose-rate 
Table 3. Laboratory tests before and 30 days after the procedure

\begin{tabular}{lccccccc} 
& AST & ALT & TBIL & ALB & WBC & PLT \\
\hline Before & $59.92 \pm 25.56$ & $53.08 \pm 12.99$ & $19.97 \pm 3.24$ & $39.34 \pm 3.68$ & $7.00 \pm 1.27$ & $227.25 \pm 59.34$ & $11.7 \pm 1.75$ \\
\hline After & $53.42 \pm 25.06$ & $49.75 \pm 14.77$ & $18.78 \pm 2.56$ & $39.84 \pm 3.44$ & $6.69 \pm 1.20$ & $221.00 \pm 51.68$ & $11.23 \pm 1.60$ \\
\hline$t$ & -0.246 & 1.015 & 1.508 & -0.841 & 1.321 & 0.556 & 0.574 \\
\hline$p$ & 0.810 & 0.332 & 0.16 & 0.418 & 0.213 & 0.144
\end{tabular}

(HDR) radiotherapy [18] In addition, stereotactic radiotherapy for body tumors (SBRT) has more and more clinical applications due to its good efficacy and low side effects. SBRT is based on the characteristics, location, and size of the tumor, using different radiotherapy segmentation modes [19]. However, the optimal separation mode and the dose limit of OAR are still unclear and require a lot of research and exploration. Therefore, ${ }^{125}$ I seed radiotherapy is an ideal method for PDAC treatment and pain control. However, the safety of these treatments, conducted via percutaneous implantation guided by CT or EUS [20,21], is still under a debate, because of a damage to structures adjacent to the pancreas, such as the liver, stomach, intestines, spleen, kidney, and surrounding blood vessels [22].

Implantation of ${ }^{125} \mathrm{I}$ seed strand and metal stent was reported to treat the main portal vein tumor thrombus by Luo et al. $[10,11]$. This new approach was demonstrated to prolong the overall survival and control tumor thrombus in the main portal vein effectively. Similarly, the SV or SMV, as branches of the portal vein that are prone to invasion by advanced PDAC, can also receive ${ }^{125} \mathrm{I}$ seed strand implantation and stent placement to treat tumor adjacent or invaded tissues.

In this study, we found that EVBT with ${ }^{125} \mathrm{I}$ seed strand implantation and stent placement present various advantages for advanced PDAC invading the SV or SMV. First, delivering ${ }^{125} \mathrm{I}$ seed strands through a patent second-order intrahepatic portal vein branch could avoid the potentially serious adverse events related to direct puncturing of the pancreas for radioactive seed implantation. Second, because the number of ${ }^{125}$ I planned for implantation was determined by the length of obstructed vessels, and radioactive seeds were linearly aligned and sealed into a 4-F catheter continuously to construct a ${ }^{125} \mathrm{I}$ seed strand, radioactive seed dislodgment after implantation could be prevented, and a longitudinal coverage of the target lesion by the radiation from ${ }^{125}$ I seeds may be achieved. Third, when tumor invasion of the SV occurs, the blood flow of SV can be restored and the pressure of distal SV can be effectively reduced with stent placement, thus preventing the occurrence of pancreatogenic segmental portal hypertension and its complications, such as upper gastrointestinal hemorrhage.

In this study, the median survival was $10.7 \pm 1.4$ months, and the disease control rate was $83.3 \%$ among all patients. The volume and activity of tumors were significantly reduced. Meanwhile, no grade 3 or 4 adverse events related to the procedure occurred within 180 days after the procedure. These results indicate that EVBT combined with stent and RAIC or TACE is a safe and feasible treatment approach for PDAC.

This study has some limitations. First, the scope of dose distribution was limited. For tumors with large radius exceeding the effective radius of ${ }^{125} \mathrm{I}$ seed $(17 \mathrm{~mm})$, the curative effect would be significantly reduced. Second, a control group was not included. Third, the sample size was too small to generate a conclusive result. Finally, a combination of chemotherapy and radiotherapy was used in the study, so it cannot be determined whether chemotherapy influences the results of this experiment. Nonetheless, our study demonstrated the effect of EVBT using ${ }^{125}$ I seed strand as a treatment modality in patients with locally advanced pancreatic duct adenocarcinoma complicated by vascular invasion. A randomized control study with an adequate sample size is warranted in the future.

\section{Conclusions}

Intraluminal brachytherapy using ${ }^{125}$ I strand may be considered as a safe and effective treatment option for patients with locally advanced PDAC complicated by vascular invasion.

\section{Funding}

This study was supported by Clinical Research Special Fund of Zhongshan Hospital, Fudan University (2016ZSLC17) and Young Teachers of Jining Medical College (JY2016KJ014).

\section{Ethic approves}

This prospective single-arm pilot study was approved by the ethics committee and institutional review board of Tengzhou Central People's Hospital.

\section{Disclosure}

The authors report no conflict of interest.

\section{References}

1. Jemal A, Bray F, Center MM et al. Global cancer statistics. CA Cancer J Clin 2011; 61: 69-90.

2. Hidalgo M. Pancreatic cancer. N Engl J Med 2010; 362: 16051617.

3. GBD 2013 Mortality and Causes of Death Collaborators. Global, regional, and national age-sex specific all-cause and cause-specific mortality for 240 causes of death, 1990-2013: a systematic analysis for the Global Burden of Disease Study 2013. Lancet 2015; 385: 117-171. 
4. Cameron JL, Riall TS, Coleman J, Belcher KA. One thousand consecutive pancreaticoduodenectomies. Ann Surg 2006; 244: $10-15$.

5. Dobelbower RR, Montemaggi P. Brachytherapy for pancreatic cancer: a review. Hepatogastroenterology 1996; 43: 333-337.

6. Willett CG, Safran H, Abrams RA et al., Gastrointestinal Committee of the Radiation Therapy Oncology Group. Clinical research in pancreatic cancer: the Radiation Therapy Oncology Group trials. Int J Radiat Oncol Biol Phys 2003; 56 (4 Suppl): 31-37.

7. Takacsi-Nagy Z, Varga J, Poller I et al. Successful treatment of a T1 cancer of the pancreatic head with high dose rate brachytherapy and external radiotherapy. Hepatogastroenterology 2002; 49: 844-846.

8. Yang M, Yan Z, Luo J et al. A pilot study of intraluminal brachytherapy using (125)I seed strand for locally advanced pancreatic ductal adenocarcinoma with obstructive jaundice. Brachytherapy 2016; 15: 859-864.

9. Huguet F, Girard N, Guerche CS et al. Chemoradiotherapy in the management of locally advanced pancreatic carcinoma: a qualitative systematic review. J Clin Oncol 2009; 27: 2269-2277.

10. Luo J, Yan Z, Liu Q et al. Endovascular placement of iodine-125 seed strand and stent combined with chemoembolization for treatment of hepatocellular carcinoma with tumor thrombus in main portal vein. J Vasc Interv Radiol 2011; 22: 479-489.

11. Luo JJ, Zhang ZH, Liu QX et al. Endovascular brachytherapy combined with stent placement and TACE for treatment of HCC with main portal vein tumor thrombus. Hepatol Int 2016; 10: 185-195.

12. Lencioni R, Llovet JM. Modified RECIST (mRECIST) assessment for hepatocellular carcinoma. Semin Liver Dis 2010; 30: 52-60.

13. Jeong SW, Jang JY, Shim KY et al. Practical effect of sorafenib monotherapy on advanced hepatocellular carcinoma and portal vein tumor thrombosis. Gut Liver 2013; 7: 696-703.

14. Ducreux M, Boige V, Malka D. Treatment of advanced pancreatic cancer. Semin Oncol 2007; 34 (2 Suppl 1): S25-30.

15. Mulcahy MF, Wahl AO, Small W. The current status of combined radiotherapy and chemotherapy for locally advanced or resected pancreas cancer. J Natl Compr Canc Netw 2005; 3: 637-642.

16. Yip D, Karapetis C, Strickland A et al. Chemotherapy and radiotherapy for inoperable advanced pancreatic cancer. $\mathrm{Co}^{-}$ chrane Database Syst Rev 2006; 3: CD002093.

17. Hong GB, Zhou JX, Sun HB et al. Continuous transarterial infusion chemotherapy with gemcitabine and 5-Fluorouracil for advanced pancreatic carcinoma. Asian Pac J Cancer Prev 2012; 13: 2669-2673.

18. Tisseverasinghe $\mathrm{S}$, Crook J, arnquist B et al. Phase II randomized pilot comparing HDR and LDR monotherapy for intermediate risk prostate cancer: report of dose escalation to dominant intraprostatic lesions. Brachytherapy 2018; 17: S20-S21.

19. Goodman KA, Kavanagh BD. Stereotactic body radiotherapy (SBRT) for liver metastases. Semin Radiat Oncol 2017; 27: 240246.

20. Yu YP, Yu Q, Guo JM et al. Effectiveness and security of CTguided percutaneous implantation of (125)I seeds in pancreatic carcinoma. Br J Radiol 2014; 87: 20130642.

21. Wang KX, Jin ZD, Du YQ et al. EUS-guided celiac ganglion irradiation with iodine-125 seeds for pain control in pancreatic carcinoma: a prospective pilot study. Gastrointest Endosc 2012; 76: 945-952.

22. Mohiuddin M, Rosato F, Barbot D et al. Long-term results of combined modality treatment with I-125 implantation for carcinoma of the pancreas. Int J Radiat Oncol Biol Phys 1992; 23: 305-311. 